\title{
A WALKTHROUgh REMOTE VIEW SYSTEM WITH AN OMNIDIRECTIONAL CAMERA
}

\author{
Chao Liu ${ }^{1}$, Toshiya Watanabe ${ }^{1}$, Susumu Shibusawa ${ }^{2}$ and Tatsuhiro Yonekura ${ }^{2}$ \\ ${ }^{1}$ Graduate School of Science and Engineering, Ibaraki University, Hitachi City, Japan \\ $\{10$ nd303r, 12nd307a\} @hcs.ibaraki.ac.jp \\ ${ }^{2}$ College of Engineering, Ibaraki University, Hitachi City, Japan \\ \{sibusawa, yone\}@mx.ibaraki.ac.jp
}

\begin{abstract}
A remote view system is a system for the delivery and presentation of information such as video and audio to users in remote locations. Methods for the construction of virtual environments based on video information have been applied to remote view technology. When a system that supports remote viewing is applied to situations such as viewing scenery or engaging in collaborative work, it is useful to provide users with a function that enables them to walkthrough a sense of immersion in a remote location environment. Although walkthroughs are possible in remote view systems that use pre-prepared images to construct virtual environments, these systems are lacking in terms of real-time performance. In this study, we built a virtual walkthrough environment and created a system that allows multiple users view images sent in real time from an omnidirectional camera installed in a remote location. In this system, when presenting an object in the environment on a real world image, the information of the object can be shown in a separate window to the walkthrough environment if necessary. By using an improved map projection method, the system produces less image distortion than the conventional projection method. We have confirmed the walkthrough capabilities of this system, evaluated the performance of the improved map projection method, and subjected it to user trials.
\end{abstract}

\section{KEYWORDS}

Remote View, Walkthrough, Omnidirectional Camera, Panoramic Image \&Projection Method

\section{INTRODUCTION}

A remote view system is a system for the delivery and presentation of video and audio to depict the situation in remote locations. Methods for the construction of virtual environments based on video information have been applied to remote view technology. In recent years, the range of scenes to which remote view systems have been applied has been expanding to include surveillance systems, monitoring systems [17] and teleconferencing systems [4, 9]. In cases such as viewing scenery and collaborative working, it is useful to provide users with interactive features [10] and other forms of information besides video [12, 13, 14, 16]. For example, when viewing scenery or animals in a nature reserve with a remote view system, instead of offering a plain video delivery system, it would be more useful to set up a virtual environment to reproduce a remote location where users can browse through introductory information about the animals or other visible items. Also, when large numbers of workers need to share information from a remote location, it is useful to implement a virtual walkthrough environment that let the workers walkthrough in the environment constructed from the remote area and view equipment information.

So far, remote view systems have employed either a live video delivery method, or a virtual environment construction method [7]. Live video remote view systems have so far not been implemented as virtual environments with walkthrough functions for moving around in three

DOI : $10.5121 /$ ijenc.2012.4501 
dimensions, or functions for providing information about objects in these environments. Conventional remote view systems that construct virtual environments use an image rendering method $[10,11]$. This method used pre-prepared images to construct an interactive walkthrough virtual environment, but since the image processing takes time to perform, the system lacks real-time capabilities. As the performance of cameras and processing equipment improves, it is starting to become possible to use methods where virtual environments are constructed based on images sent in real time [18]. However, when using multiple unidirectional cameras, the relative positions of the cameras have to be calibrated, and this makes the system structure more complex.

On the other hand, in recent years there has been an improvement in the performance of omnidirectional cameras that can capture an entire $360^{\circ}$ range in a single image, and these are being applied to live video systems such as surveillance systems [15] and teleconferencing systems [9]. Video pictures from an omnidirectional camera can be used without limiting the number of users, and are suitable for remote viewing with large numbers of users. Therefore, in this study we created a remote view system that uses an omnidirectional video camera to allow multiple users to perform walkthroughs in different directions. Using a method that improves on the conventional map projection method, the omnidirectional image captured by the omnidirectional camera is projected into a panoramic image which is presented to the users. This system also has a function that uses images and audio to describe objects in the environment, allowing it to provide users with information besides the actual video.

In this paper, we confirmed the walkthrough function of this system, we evaluated the performance of the improved map projection method, and we subjected the system to user trials. By comparing with images captured with a unidirectional camera from the same viewpoint as the omnidirectional camera, we confirmed that the improved map projection method results in less distortion than the conventional map projection method. We also confirmed the interactive walkthrough functions whereby users can navigate using a mouse and keyboard, and the function for presenting descriptive audio and images corresponding to the user's position. We also performed user trials to evaluate attributes such as system delays.

Section 2 of this paper introduces the related research and the background to this study. Section 3 describes the walkthrough image generation method, and Section 4 explains the design of the walkthrough system. In Section 5, we discuss the implementation of this system, and in Section 6, we describe our experiments and discuss the results. Finally, in Section 7 we conclude with a summary and a discussion of topics for further study.

\section{RELATED RESEARCH}

Hitherto, most remote view systems have used either a live video delivery method or a virtual environment method.

\subsection{Live video remote view system}

A remote view system that uses live video is able to deliver video to users from diverse types of camera. It is possible to use multiple PTZ (pan, tilt, and zoom) cameras so that multiple users can look in different directions by freely operating their own individual cameras, but the number of users that can be accommodated is restricted to the number of PTZ cameras [17]. Although it is possible to accommodate additional users by using algorithms that satisfy as many users as possible, this approach is not compatible with several tens of users. Remote view systems that use omnidirectional cameras $[9,15]$ are applied to live video systems for purposes such as tourism promotion, surveillance and videoconferencing. In this sort of system, the omnidirectional image is projected into a panoramic image, and depending on the user's 
requests, part or all of the video is presented to the user. However, since a virtual environment is not constructed, this system does not have a complete set of walkthrough functions that allow movement in the forward/backward, up/down and left/right directions.

\subsection{Virtual reality remote view system}

Virtual reality is a technique that involves building a virtual environment and presenting it to users instead of their real environment $[1,2,5,6]$. An image rendering technique is used to generate images from a new viewpoint based on the captured images [8]. Research has been carried out into using this technique to construct a remotely viewable virtual environment from large numbers of pictures captured with unidirectional cameras [3]. The use of real images means that the generated environment closely resembles the real world. However, it is difficult to generate real-time virtual environments because time-consuming preliminary work is needed to prepare images for the construction of a walkthrough environment.

Also, when constructing a virtual environment using real-time images from multiple unidirectional cameras, the system construction is made complicated due to the need for relative positioning and calibration of the cameras.

\section{WALKTHROUGH IMAGE GENERATION METHOD}

This section presents the detail description of the walkthrough image processing methods: improved map projection method and walkthrough image generation method.

\subsection{An improved map projection method}

Images captured with an omnidirectional camera are circular in shape. Figure 1 shows a photograph of an omnidirectional camera. The angle $\alpha$ in this Figure shows the range of view at the side of the omnidirectional camera. Figure 2 shows a circular image captured by this omnidirectional camera.

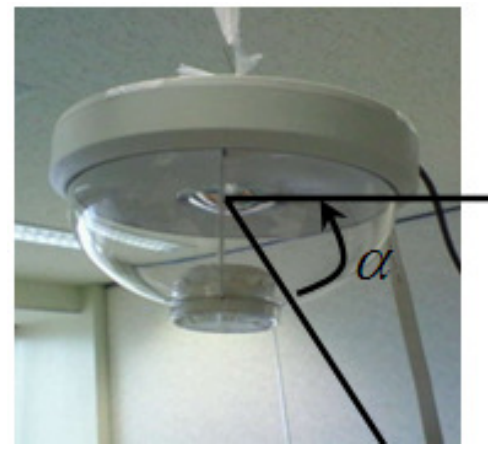

Figure 1. Omnidirectional camera Figure 2. Image captured by the omnidirectional camera

Figure 3 shows the coordinate system of the circular omnidirectional image. The circular image is centered at $\left(x_{0}, y_{0}\right)$ with a radius of $R$, and it is assumed that $x_{0}=y_{0}=R$. If an arbitrary point $P$ in this image has coordinates $(x, y)$, its distance from the center of the circle is $r$, and this radius subtends an angle $\beta$ from the $x$ axis, then the coordinates $(x, y)$ of point $P$ satisfy the following relationships:

$$
\begin{aligned}
& x=x_{0}+r \cos \beta \\
& y=y_{0}+r \sin \beta
\end{aligned}
$$




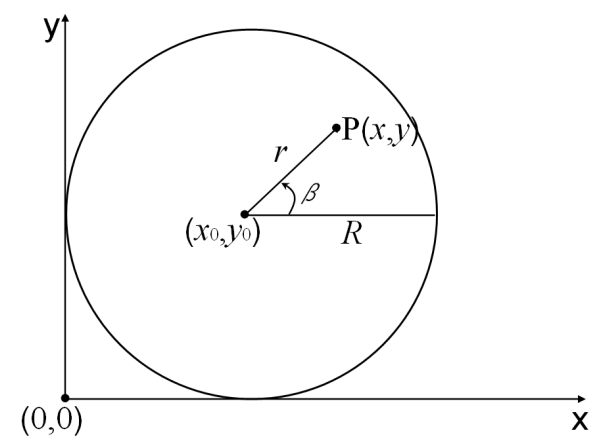

Figure 3. Coordinates of a circular omnidirectional image

A map projection method is used to project the circular image into a panoramic image. The map projection method transforms a pixel at point $\mathrm{P}(x, y)$ in the omnidirectional image into a point $\mathrm{P}^{\prime}$ at coordinates ( $\left.\mathrm{x}^{\prime}, \mathrm{y}^{\prime}\right)$ in the Cartesian coordinate system. The point $\left(x^{\prime}, y^{\prime}\right)$ satisfies the following equations:

$$
\begin{aligned}
& x^{\prime}=R(2 \pi-\beta) \\
& y^{\prime}=r
\end{aligned}
$$

Figure 4 shows the principle of the map projection method. For the specified region $S$ of the omnidirectional image in Figure 4(a) and (b), Figure 4(c) shows the image projected using the map projection method shown in Equations (3) and (4). Also, Figure 5(a) and (b) show a representation of a specified region of the omnidirectional image divided into $\mathrm{n}$ sections in the radial direction, and the panoramic image representation obtained by projecting this region using the map projection method. In Figure 5(a), the sector closest to the center of the circular image (sector 1) contains no image data. Figure 5(c) shows a panoramic image obtained by the improved map projection method described below. In the improved map projection method, the height of each region is altered according to its distance from the center of the circular image. The symbols used in Figures. 4 and 5 are described in Table 1.
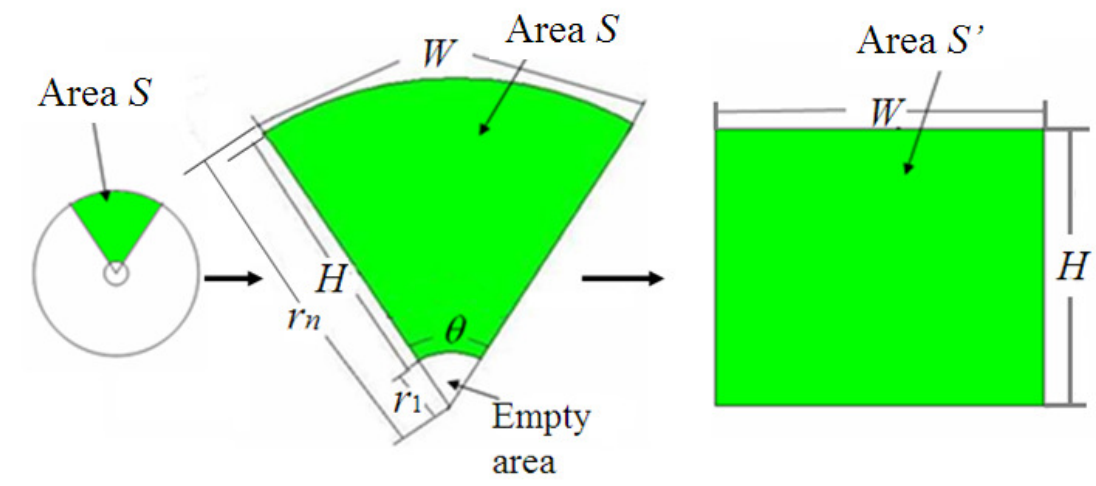
(a) Omnidirectional image
(b) Part of omnidirectional image
(c) Panoramic image

Figure 4. Principle of map projection 


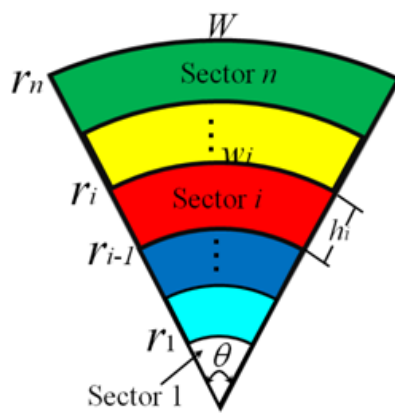

(a) Omnidirectional image

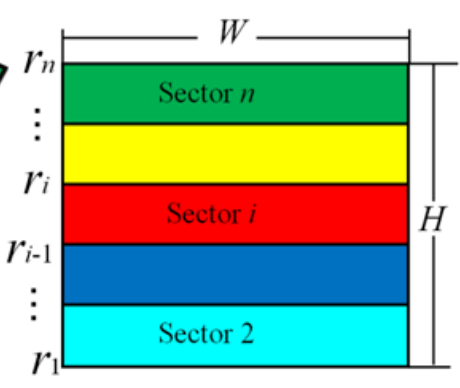

(b) Map panoramic image

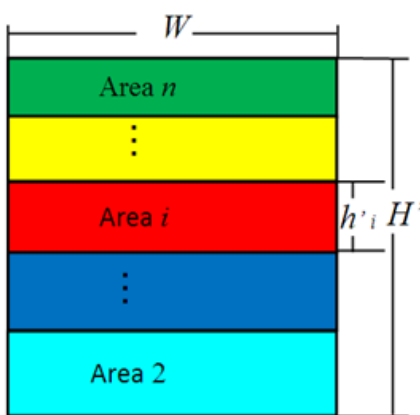

(c) Improved map panoramic image

Figure 5. Partitioning of image into sectors

Table 1. Variable definitions.

\begin{tabular}{|l|l|}
\hline Variable & Definition \\
\hline$S$ & Specified region of omnidirectional image \\
\hline$S^{\prime}$ & Corresponding region in panoramic image \\
\hline$\theta$ & Angle of region $S$ \\
\hline$r_{i}$ & Radius of sector $i$ \\
\hline$w_{i}$ & Arc length of sector $i$ \\
\hline$h_{i}$ & Height of sector $i$ \\
\hline$W$ & Arc length of region $S$ and width of region $S^{\prime}$ \\
\hline$H$ & Height of region $S^{\prime}$ \\
\hline$h_{i}^{\prime}$ & Height of region i of improved panoramic image \\
\hline$H^{\prime}$ & Height of improved panoramic image \\
\hline
\end{tabular}

The width $W$ and height $H$ of the rectangle in Figure 5(b) satisfy the following formulae:

$$
\begin{aligned}
& W=\theta r_{n}=\theta R \\
& H=r_{n}-r_{1}
\end{aligned}
$$

The arc length $w_{i}$ of sector $i$ in Figure 5(a) is given by the following formula:

$$
w_{i}=\theta r_{i}
$$

When projecting a circular image using the map projection method shown in Equations (3) and (4), the pixels of the circular image are stretched to form a panoramic image. When forming the panoramic image shown in Figure 5(b), for an arbitrary sector $i$, the scale factor $m_{i}$ of stretching in the horizontal direction is given by the following formula:

$$
m_{i}=\frac{W}{w_{i}}
$$


International Journal of Computer Networks \& Communications (IJCNC) Vol.4, No.5, September 2012

Substituting Equations (5) and (7) into Equation (8), the scale factor $m_{i}$ comes out as follows:

$$
m_{i}=\frac{R}{r_{i}}
$$

When the map projection method is used to project a circular image, pixels in the horizontal direction of sector $i$ are scaled up by a factor of $m_{i}$, so the ratio of the vertical and horizontal image dimensions changes, leading to distortion. In the improved map projection method, the height $h_{i}$ is therefore scaled up by a factor of $m_{i}$ in sector $i$ of the panoramic image obtained by the original map projection method.

$$
h_{i}^{\prime}=m_{i} h_{i}=\frac{R}{r_{i}} h_{i}
$$

The height $H^{\prime}$ of the improved panoramic image is expressed as follows:

$$
H^{\prime}=\sum_{i=2}^{n} m_{i} h_{i}=R \sum_{i=2}^{n} \frac{h_{i}}{r_{i}}
$$

Since the improved map projection method preserves the ratio of vertical and horizontal dimensions of objects in the image, it is able to generate images with less distortion.

\subsection{Generating walkthrough images}

A walkthrough image is an image that represents the field of view of a user moving through a virtual space. When the user moves forward, the field of view becomes narrower, but objects within this field of view become larger. When the user's viewpoint moves in the left/right or up/down direction, the range of the field of view changes, but objects within the field of view stay the same size. Figures 6 and 7 show some examples of how the user's field of view changes due to movement of the user within a virtual space and due to changes of viewpoint, respectively. Figure 6 shows an example of the walkthrough image area changes as the user moves forward, and Figure 7 shows some examples of the walkthrough image area changes as the user's viewpoint moves.

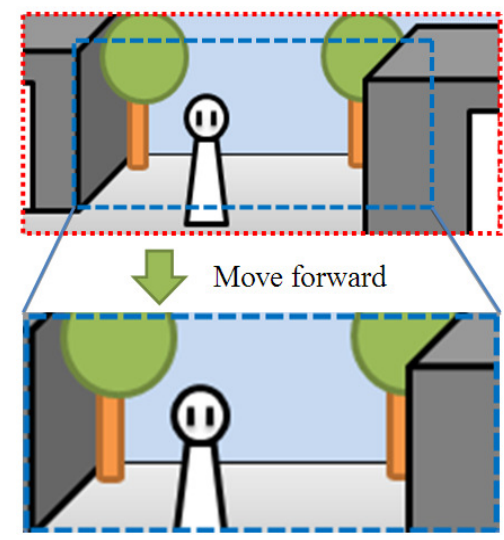

Figure 6. Example of forward movement 


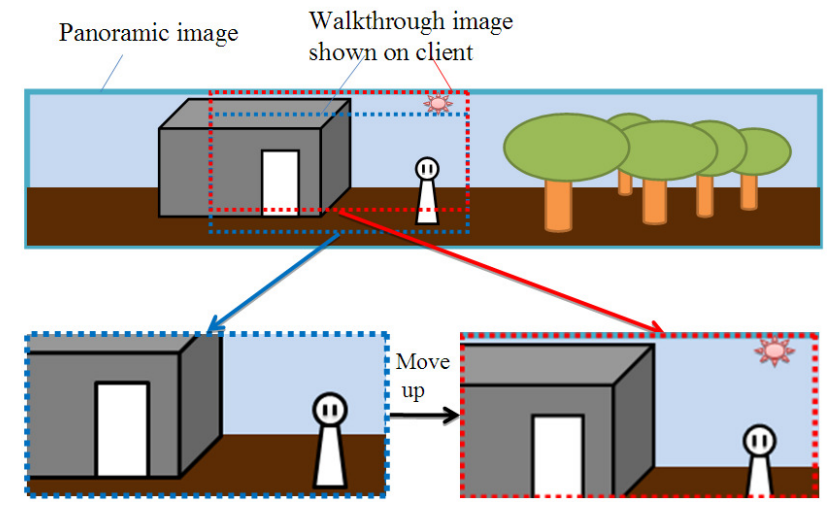

(a) Viewpoint moving upwards

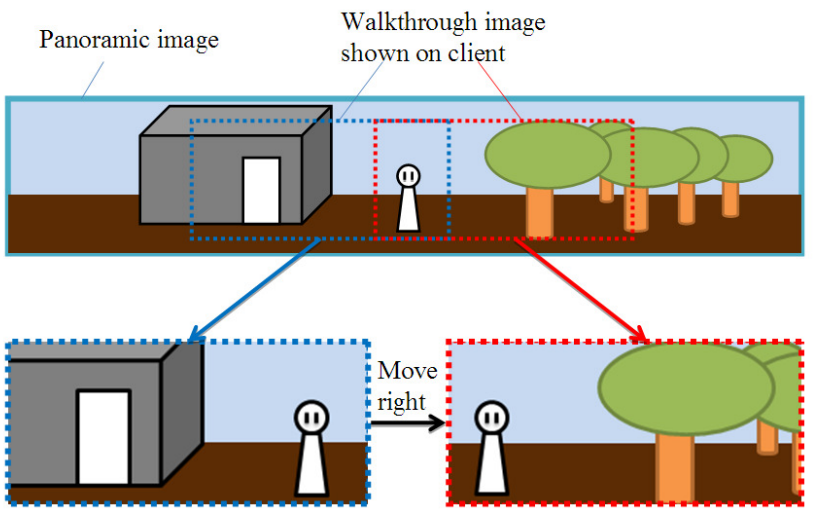

(b) Viewpoint moving to the right

Figure 7. Examples of changing viewpoints

In Figure 8, the rectangle $A B C D$ represents the entire panoramic image. Rectangle $A_{1} B_{1} C_{1} D_{1}$ represents the range of the walkthrough image before the user moves forward or changes the viewpoint to the right, and $\mathrm{A}_{2} \mathrm{~B}_{2} \mathrm{C}_{2} \mathrm{D}_{2}$ represents the range of the image after each of these movements. The coverage of the omnidirectional camera extends over $360^{\circ}$, while in the vertical direction, the angle of elevation covers the range from the angle of elevation to the angle of dip. Consequently, the range of the walkthrough images depends on the camera parameters.

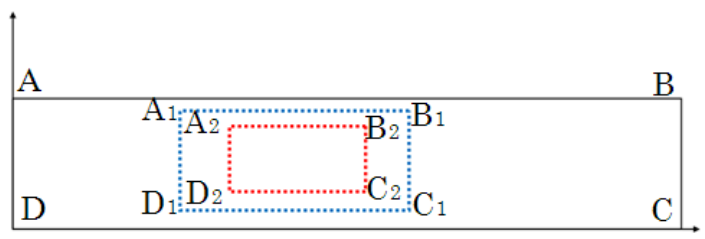

(a) Forward movement

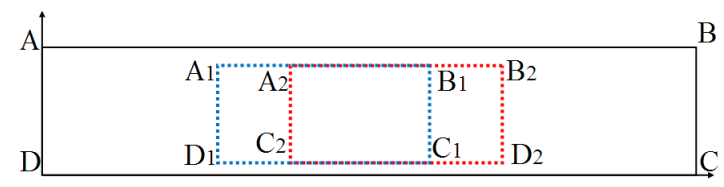

(b) Viewpoint moving to the right

Figure 8. Image regions before and after walkthrough movements 
International Journal of Computer Networks \& Communications (IJCNC) Vol.4, No.5, September 2012

In Figure 8, the panoramic mapped image $\mathrm{ABCD}$ consists of $L \times H$ pixels, and the rectangular portion $\mathrm{A}_{1} \mathrm{~B}_{1} \mathrm{C}_{1} \mathrm{D}_{1}$ that is presented to the user comprises $l \times h$ pixels. Points $\mathrm{D}$ and D1 are assigned coordinates of $(0,0)$ and $(i, j)$ respectively. The pixel at an arbitrary point $\mathrm{P}(x, y)$ on the panoramic mapped image $\mathrm{ABCD}$ is represented by $\left(a_{x}, b_{y}\right)$. The set $K$ of pixel values in the panoramic mapped image $\mathrm{ABCD}$ is represented by the following matrix.

$$
K\left(a_{x}, b_{y}\right)=\left[\begin{array}{ccccc}
\left(a_{0}, b_{H-1}\right) & \left(a_{1}, b_{H-1}\right) & \left(a_{2}, b_{H-1}\right) & \cdots & \left(a_{L-1}, b_{H-1}\right) \\
\vdots & \vdots & \vdots & \ddots & \vdots \\
\left(a_{0}, b_{2}\right) & \left(a_{1}, b_{2}\right) & \left(a_{2}, b_{2}\right) & \cdots & \left(a_{L-1}, b_{2}\right) \\
\left(a_{0}, b_{1}\right) & \left(a_{1}, b_{1}\right) & \left(a_{2}, b_{1}\right) & \cdots & \left(a_{L-1}, b_{1}\right) \\
\left(a_{0}, b_{0}\right) & \left(a_{1}, b_{0}\right) & \left(a_{2}, b_{0}\right) & \cdots & \left(a_{L-1}, b_{0}\right)
\end{array}\right] \quad(0 \leq x<L, 0 \leq y<H)
$$

In Figure 8, the sets of pixel values in $\mathrm{A}_{1} \mathrm{~B}_{1} \mathrm{C}_{1} \mathrm{D}_{1}$ and $\mathrm{A}_{2} \mathrm{~B}_{2} \mathrm{C}_{2} \mathrm{D}_{2}$ are both subsets of set $K$. The set of pixel values in image $A_{1} B_{1} C_{1} D_{1}$ before the walkthrough movement is expressed by the following matrix.

$$
M=K\left(a_{x}, b_{y}\right) \quad(i \leq x<i+l, j \leq y<j+h)
$$

When the user moves forward and backward as shown in Figure 8(a), a new image is generated. In the case of a forward movement, the user's field of view becomes narrower, and the range of the image becomes correspondingly smaller. If $\Delta h$ is the reduction of the vertical dimension and $\Delta l$ is the reduction of the horizontal dimension, the set of pixel values used in the walkthrough image after the movement is as follows:

$$
N=K\left(a_{x}, b_{y}\right) \quad(i+\Delta l / 2 \leq x<i+l-\Delta l / 2, j+\Delta h / 2 \leq y<j+h-\Delta h / 2)
$$

Due to the change in the set of pixel values shown in Equation (13), the image presented to the user changes in size. When the user has moved forward and backward, the image's change of scale $\delta$ is expressed as follows:

$$
\delta=\frac{l \times h}{(l-\Delta l)(h-\Delta h)} \quad(\Delta l<l, \Delta h<h)
$$

For expansions and contractions of the image size, the values of $\Delta h$ and $\Delta l$ are negative and positive respectively. As shown in Figure 8(b), the range of the walkthrough image changes when the user changes the viewpoint in the up/down or left/right direction. Since the walkthrough image is part of a panoramic image, the limits on changes to the range of the image range depend on the size of the walkthrough image. When the viewpoint changes by $\Delta h$ ' in the vertical direction and $\Delta l^{\prime}$ in the horizontal direction, the set $Q$ of pixel values included in the image $A_{2} B_{2} C_{2} D_{2}$ after the change of viewpoint is as follows:

$$
Q=K\left(a_{x}, b_{y}\right) \quad\left(i+\Delta l^{\prime} \leq x<i+l+\Delta l^{\prime}, j+\Delta h \leq y<j+h+\Delta h^{\prime}\right)
$$

When the viewpoint changes in an upward direction, the value of $\Delta h^{\prime}$ is positive, and when the viewpoint changes in a downward direction, the value of $\Delta h$ ' is negative. When the viewpoint changes in a rightward direction, the value of $\Delta l^{\prime}$ is positive, and when the viewpoint changes in a leftwards direction, $\Delta l^{\prime}$ is negative. For a change in the user's viewpoint, the set of pixel values $Q$ is presented to the user as the walkthrough image. 


\section{DESIGN OF REMOTE VIEW SYSTEM}

This system consists of a server, a client and an omnidirectional camera. The system configuration is shown in Figure 9. An image from a remote location is captured by the omnidirectional camera, and a server uses the improved map projection method to project the omnidirectional image into a panoramic image, which is transmitted to the client. The client then constructs a virtual environment from the panoramic image sent from the server together with pre-prepared images and audio describing objects that appear in this image.

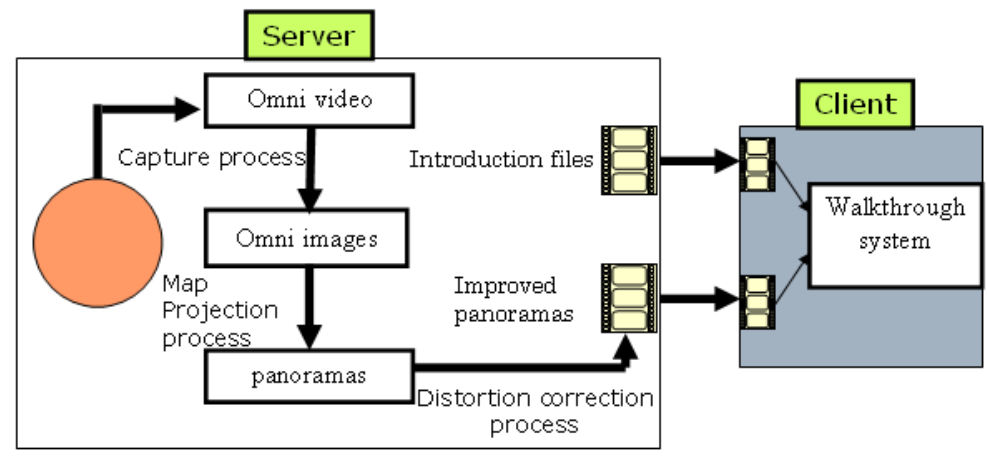

Figure 9. Configuration of the remote view system

The walkthrough virtual environment is constructed as follows:

(1) The client connects to the server, and downloads the descriptive files for one time.

(2) The client receives panoramic images from the server in real time.

(3) The client uses the descriptive audio and image files together with the panoramic images sent in real time to construct a virtual environment that users can walk through freely.

Table 2 shows the functions of each constituent part of the system. When a user gets sufficiently close to objects in the panoramic image, the system presents the pre-prepared images and audio to the user. When the system presents a virtual environment to the user based on real images, instead of replacing the images of objects with virtual objects, the descriptive images and audio are presented on a separate screen according to the user's requests.

Table 2. The functions of files used in the virtual environment system.

\begin{tabular}{|l|l|}
\hline Type of constituent part & Function \\
\hline Panoramic image & $\begin{array}{l}\text { Image used for the construction of a } \\
\text { walkthrough environment }\end{array}$ \\
\hline $\begin{array}{l}\text { Descriptive images and } \\
\text { audio }\end{array}$ & $\begin{array}{l}\text { Audio and images describing objects in } \\
\text { the virtual environment }\end{array}$ \\
\hline
\end{tabular}

\section{1) Panoramic images for walkthrough}

The server uses the improved map projection method to project images acquired from the omnidirectional camera into a panoramic image and sent the panoramic image to the client. The client builds a virtual walkthrough environment based on this panoramic image and the descriptive files. When the user issues a movement command, the client extracts and displays part of the panoramic image according to the user's commands. 
International Journal of Computer Networks \& Communications (IJCNC) Vol.4, No.5, September 2012

\section{2) Descriptive audio and images}

When the distance from the center of the walkthrough image to a specific object falls below a certain level, it is judged that the user has approached this object in the virtual environment, and the client presents the descriptive audio and images to the user. At this time, instead of replacing the image of the object with a virtual object, the images and audio are presented to the user on a separate screen according to the user's request. The client downloads the descriptive audio and images from the server when the client first connects to the server.

\section{3) Walkthrough input method in the virtual environment}

The system uses a mouse and keyboard to perform walkthrough actions. By operating the keyboard, the user is able to move up, down, forward, backward, left and right within the virtual environment. When the user clicks the mouse on a target location in the screen, the system calculates the distance between the central point of the screen and the target location, and automatically moves to the target location.

\section{SYSTEM IMPLEMENTATION}

This virtual environment system is configured from a server and a client. Table 3 lists the parameters of the operating environment used for this system.

Table 3. Operating environment of walkthrough system.

\begin{tabular}{|l|l|l|}
\hline & Server & Client \\
\hline CPU & $\begin{array}{l}\text { Intel Core 2 Quad Q9550 } \\
\text { @ 2.6 GHZ 2.7 GHZ }\end{array}$ & $\begin{array}{l}\text { Intel Core 2 Duo E6550 } \\
\text { @ 2.3 GHz 2.3 GHz }\end{array}$ \\
\hline Flash Memory & 4 GB & 1 GB \\
\hline OS & Windows Vista & Windows XP \\
\hline
\end{tabular}

This system was developed using the OpenCV image processing library, with Visual $\mathrm{C}++$ as a development environment. The image acquisition rate of the omnidirectional camera is $5 \mathrm{fps}$, so the rate at which the client acquires images from the server was correspondingly set to $5 \mathrm{fps}$. After the system starts up, the server captures a $1632 \times 1216$ pixel omnidirectional image, and uses the map projection method to project it into a $3818 \times 608$ pixel panoramic image. After that, the improved map projection method is used to obtain a $3818 \times 906$ pixel panoramic image. The image processing time is kept to within 0.2 seconds.

\section{EXPERIMENTS AND DISCUSSION}

We performed experiments to evaluate the following aspects of this virtual environment system:

(1) Improved map projection method

(2) Walkthrough function

(3) User trials

\subsection{Results of experiments using the improved map projection method}

When the map projection method is used to convert the omnidirectional image of Figure 2, a panoramic image Figure 10 is obtained. Figure 11 shows the panoramic image obtained by using the improved map projection method to convert the same image. 
International Journal of Computer Networks \& Communications (IJCNC) Vol.4, No.5, September 2012

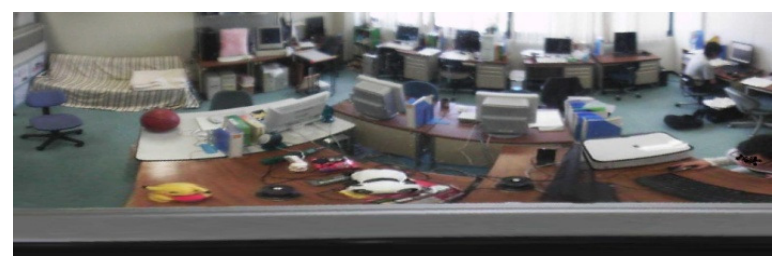

Figure 10. Panoramic image mapped by the map projection method

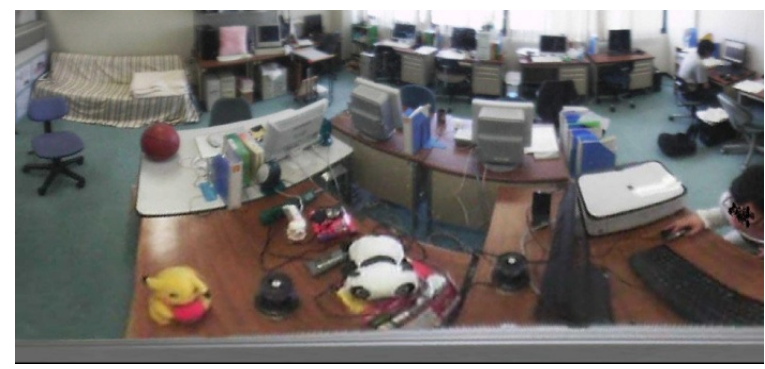

Figure 11. Panoramic image obtained by the improved map projection method

To evaluate the improved map projection method, we generated panoramic images using the conventional projection method and the improved projection method, and we measured the aspect ratios of objects in these images. Figure 12 shows some examples of panoramic images obtained by the two projection methods, together with images captured from the same angles by a unidirectional camera. From left to right, the images in this figure were obtained by the conventional map projection method, the improved map projection method, and the unidirectional camera.
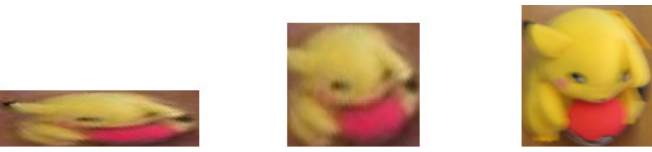

(a) Sample A
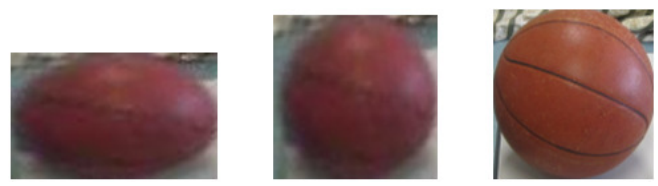

(a) Sample B
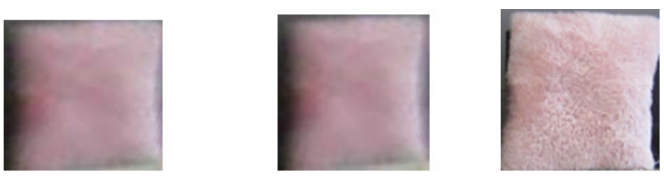

(a) Sample C

Figure 12. The appearance of objects in images obtained by three methods

We measured the aspect ratios of objects in the images obtained by the two projection methods, and we calculated the discrepancy from the aspect ratios of the images captured by the 
International Journal of Computer Networks \& Communications (IJCNC) Vol.4, No.5, September 2012

unidirectional camera. Table 4 shows the results of classifying the discrepancy according to the distance of the objects from the camera. The results show that the object images in the middle column of Figure 12 are less distorted than the object images in the left column of Figure 12, and with improved map projection method, we can obtain better quality images than conventional map projection method.

Table 4. Aspect ratio discrepancy relative to a unidirectional camera.

\begin{tabular}{|l|l|l|}
\hline \multirow{2}{*}{ Dip angle (degree) } & \multicolumn{2}{|c|}{ Difference of height/width ratio (\%) } \\
\cline { 2 - 3 } & Map projection & Improved map projection \\
\hline $10-20$ & 1 & 1 \\
\hline $40-55$ & 42 & 5 \\
\hline $70-80$ & 74 & 15 \\
\hline
\end{tabular}

\subsection{Walkthrough experimental results}

Figure 13 shows an example of the images obtained when the client's viewpoint is moved to the right at a certain point in time. Figure 13(a) shows the image displayed before the move, and Figure 13(b) shows the image displayed after a move initiated using the keyboard. The dotted lines show how the positions of an object before and after the move are different. Figure 14 shows some examples of walkthroughs performed using the mouse. Figure 14(b) shows the images obtained by performing walkthroughs to the locations shown by the two circles in Figure 14(a). The movements to the specified positions were accurately performed.

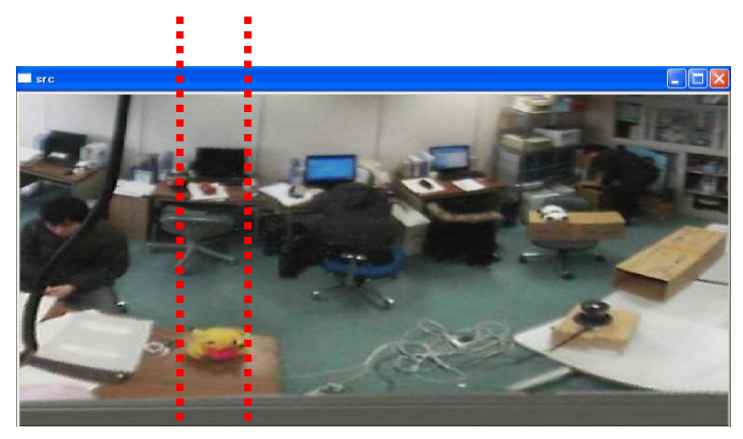

(a) Image displayed before move

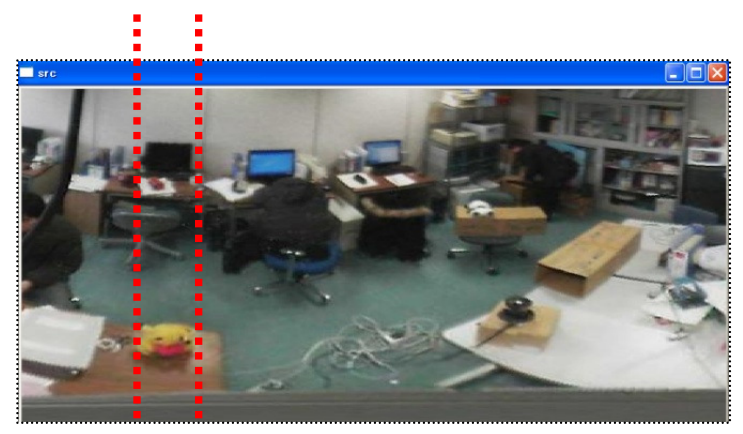

(b) Images displayed after move

Figure 13. Examples of walkthroughs performed using the keyboard 
International Journal of Computer Networks \& Communications (IJCNC) Vol.4, No.5, September 2012

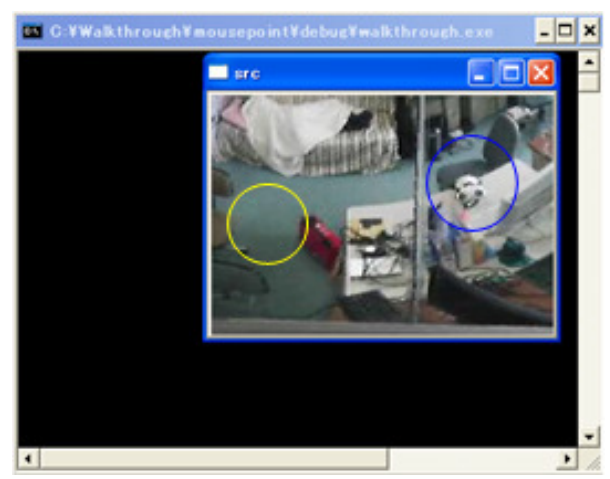

(a) the image displayed before the move
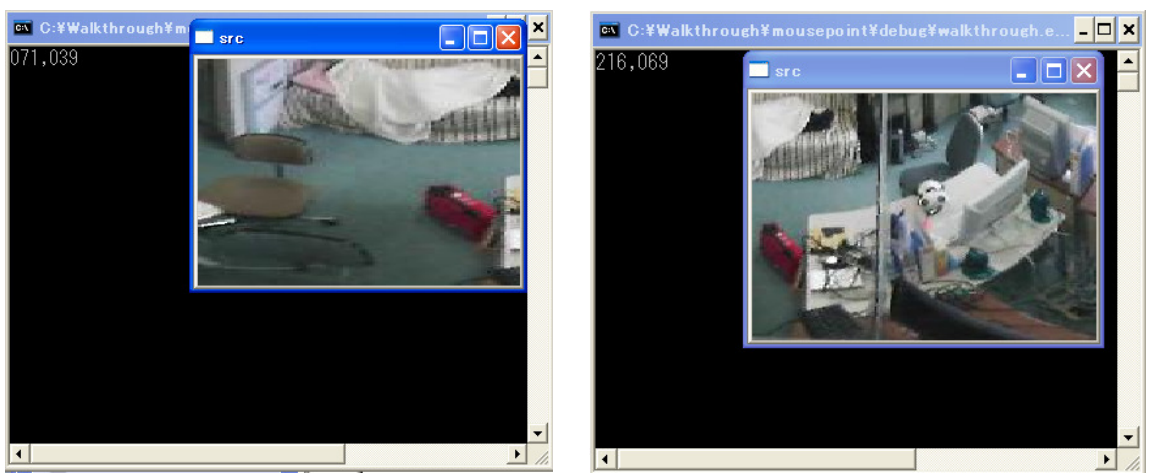

(b) Images after walkthroughs to circled locations

Figure 14. Examples of walkthroughs performed using the mouse

In this system, descriptive images and audio are automatically output when the user approaches specific locations. Figure 15 shows an example where the user has approached the black omnidirectional camera at the position of the circle in the panoramic image, whereupon the system outputs a window at the top left of the screen to describe this camera.

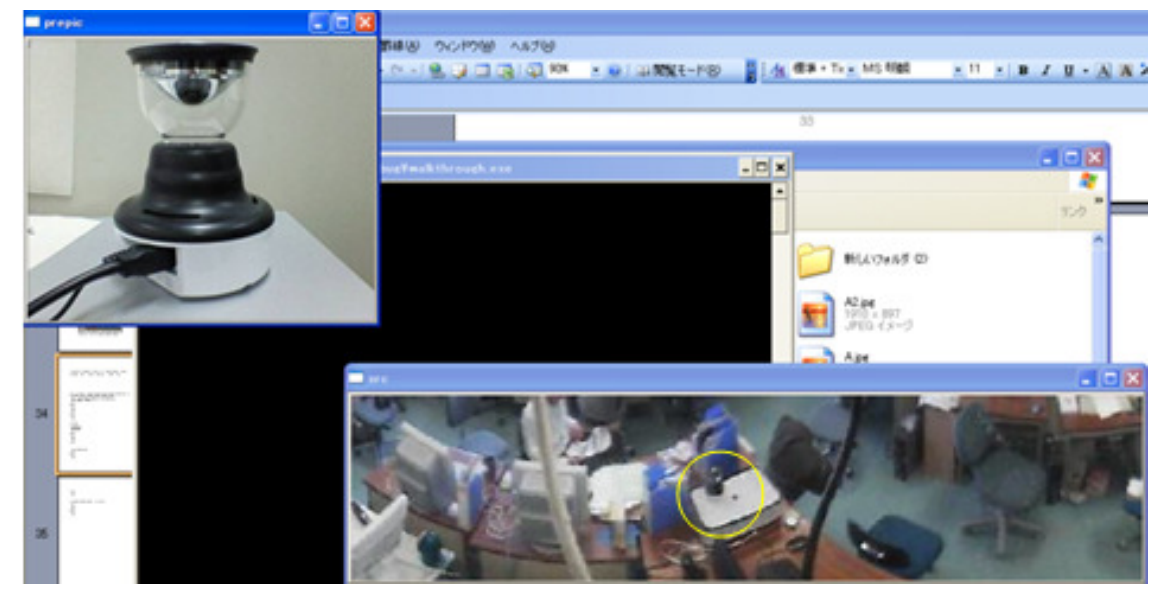

Figure 15. An example of the output of descriptive images and audio 


\subsection{User trials}

We performed user trials in which this system was used by 10 users. Figure 16 shows parts of the questionnaire results.

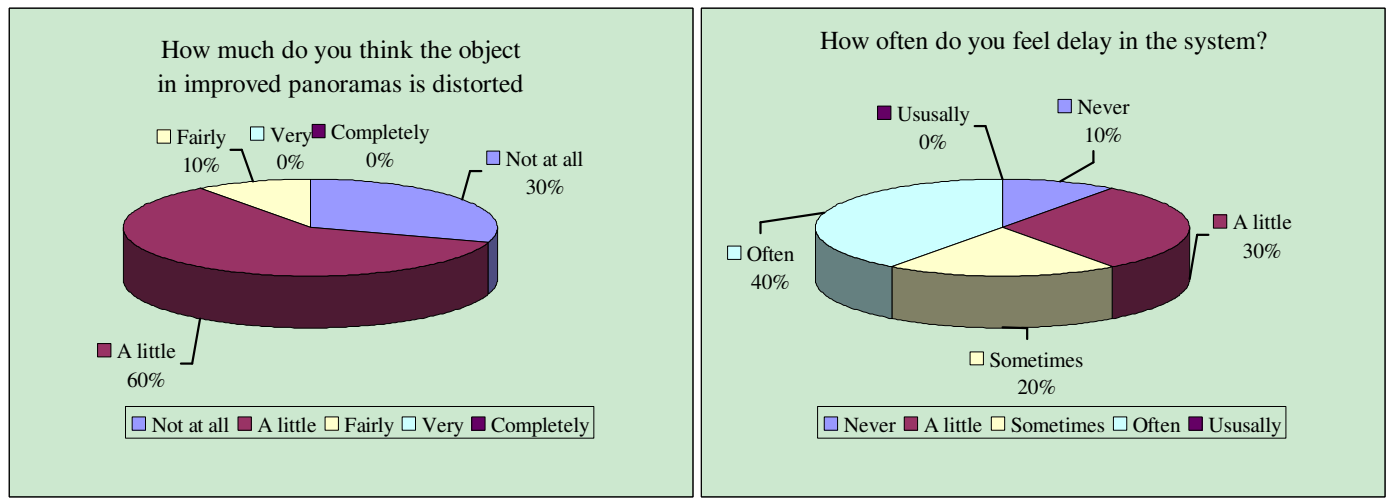

Figure 16. Parts of questionnaire results

From the questionnaire results, we found that the improved map projection method produces images that resemble the actual objects more closely than images produced by the conventional map projection method. We also found that approximately $60 \%$ of users felt some degree of delay in the walkthrough images when using this walkthrough system to move.

\section{CONCLUSION}

In this paper, we constructed a virtual environment system that supports real-time walkthroughs by using an improved map projection method to project an omnidirectional image into a panoramic image. This system consists of a server, a client, and an omnidirectional camera. The server projects the video images from the omnidirectional camera into a panoramic image, and sends this image to the client. The client uses this panoramic image together with descriptive files to build a virtual environment. In this study, we implemented a function that enables interactive walkthroughs by using a mouse and keyboard, and a function that displays descriptive audio and images according to the user's position.

The improved map projection method used in this system results in panoramic images with less aspect ratio distortion than the conventional map projection method. Also, in the questionnaire results of a user trial of the walkthrough system, some users expressed that they felt some degree of delay when operating the system. Issues for future study include conducting experiments using a more advanced omnidirectional camera, and using the system in real monitoring applications such as sightseeing and monitoring remote environments.

\section{REFERENCES}

[1] R. Azuma, Y. Baillot, R. Behringer, S. Feiner, S. Julier, \& B. MacIntyre, (2001) "Recent advances in augmented reality", IEEE Computer Graphics and Applications, Vol.21, No.6, pp.34-47.

[2] T. E. Boult, (1998) "Remote reality demonstration", IEEE Conf. on Computer Vision and Pattern Recognition, pp.966. 
International Journal of Computer Networks \& Communications (IJCNC) Vol.4, No.5, September 2012

[3] D. H. Lee \& S. K. Jung, (2003) "Capture conFigureuration for image-based street walkthroughs”, Second Int'l Conf. on Cyberworlds, pp.151-157.

[4] K. Yamazawa, T. Ishikawa, Y. Nakamura, K. Fujikawa, N. Yokoya, \& H. Sunahara, (2005) "Telepresence system using web browsers and omnidirectional video streams", IEICE Trans. on Info. and Syst., Vol.J88-D-II, No.8, pp.1750-1753.

[5] J. R. Kós, A. S. Barbosa, C. Krykhtine, E. Muniz da Silva, \& R. C. Paraizo, (2000) "The city that doesn't exist: multimedia reconstruction of Latin American cities", IEEE MultiMedia, Vol.7, No.2, pp.56-60.

[6] H. Hirayu, T. Ojika, \& R. Kijima, (2000) "Constructing the historic villages of Shirakawa-Go in virtual reality”, IEEE MultiMedia, Vol.7, No.2, pp.61-64.

[7] F. Z. Qureshi \& D. Terzopoulos, (2007) "Surveillance in Virtual Reality: System Design and Multi-Camera Control”,2007 IEEE Conference on Computer Vision and Pattern Recognition, pp.1-8.

[8] S. Ono, T. Mikami, K. Ogawara, M. Kagesawa, H. Kawasaki, \& K. Ikeuchi, (2000) “Arbitrary view position and direction rendering for large-scale scenes", IEEE Conf. on Computer Vision and Pattern Recognition, pp.296- 303.

[9] H. Ookuzu \& Y. Shibata, (2009) "Implementation and performance evaluation of a new teleconference system by GigaEther based omni directional video cameras", ICDCS 2009 Workshops, pp.374-379.

[10] D. Aliaga \&I. Carlbom, (2001) "Plenoptic stitching: A scalable method for reconstructing interactive walkthroughs", ACM SIGGRAPH, pp.443-450.

[11] L. McMillan \& G. Bishop, (1995) "Plenoptic modeling: An image-based rendering system", ACM SIGGRAPH, pp32-43.

[12] M. Rodrigues, N. Mendonça \& R. Barbosa, (2006) "Interactive mobile 3D graphics for on-thego visualization and walkthroughs", SAC '06, pp.1002-1007.

[13] G. Bruder, F. Steinicke, \& K. Hinrichs, (2009) “Arch- Explore: A natural user interface for immersive architecttural walkthroughs”, 2009 IEEE Symp. on 3D User Interfaces, pp.75-82.

[14] C. Liu, S. Shibusawa, \& T. Yonekura, (2010) "A walkthrough system with improved map projection panoramas from omni directional images", 2010 Symposia and Workshops on Ubiquitous, Autonomic and Trusted Computing, pp.45-51.

[15] T. Kon \& Y. Shibata, (2011) "Seamless surveillance system by omni directional camera network”, Int'l Conf. on Network-Based Information Systems, pp.156-161.

[16] T. C. Peck, H. Fuchs, \& M. C. Whitton, (2011) "The design and evaluation of a large-scale realwalking locomotion interface", IEEE Trans. on Visualization and Computer Graphics, Vol. 18, No. 7, pp.147-148.

[17] S. Ohno, T. Ishikawa, K. Yamazawa, \& N. Yokoya, (2010) "Video presentation system satisfying multiple users' requests using multiple PTZ cameras for child-care facility", IEICE Trans. on Info. and Syst., Vol.J93-D, No.3, pp.282-291.

[18] E. P. Affonso \& A. C. Sementille, (2007) "Support on the remote interaction for augmented reality system", Int'l Conf. on Artificial Reality and Telexistence, pp.190-196. 